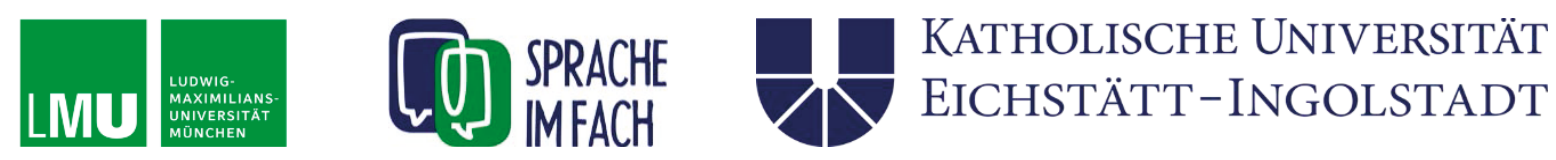

Kirstin Ulrich, Magdalena Michalak (2019)

\title{
Sprachsensibler Fachunterricht
}

\section{Definition}

Unter sprachsensiblem Fachunterricht versteht man ein didaktisches Konzept des integrierten fachlichen und sprachlichen Lernens. Die fachspezifischen Inhalte, Arbeits- und Denkweisen werden den Lernenden durch eine sprachbewusste Vorgehensweise nähergebracht. Zugleich werden ihnen die (fach-)sprachlichen Kompetenzen vermittelt, die für ihre aktive Teilnahme am Fachunterricht unabdingbar sind und ihnen die für das jeweilige Fach angemessene Anwendung der Sprache ermöglichen. Mit Fachunterricht ist dabei jeder Unterricht gemeint: Sprachenunterricht und Sachfachunterricht. Sprache und das sprachliche Handeln werden bei der Unterrichtsplanung, -vorbereitung, -durchführung und -nachbereitung konsequent berücksichtigt.

\section{Modelle integrierten Fach- und Sprachlernens}

Für derartige Herangehensweise sind in der Literatur verschiedene Bezeichnungen zu finden, die oftmals zwar synonym verwendet werden, allerdings auf unterschiedliche Schwerpunkte und Zielsetzungen hindeuten. Während „sprachsensibel“ im Sinne von Leisen (2013) beispielsweise den Einsatz von sprachfördernden Methoden insbesondere bei der Arbeit mit Lernenden nicht-deutscher Erstsprache im Unterricht der einzelnen Fächer bedeutet, hat „Durchgängige Sprachbildung“ (Gogolin, 2013) den systematischen Aufbau von bildungssprachlichen Kompetenzen fächerübergreifend in allen pädagogischen Institutionen zum Ziel. Daran anknüpfend werden im „sprachbildenden Fachunterricht“ (Riebling, 2013) die erforderlichen sprachlichen Mittel an alle Schüler(innen) vermittelt. Wird der Fachunterricht „sprachbewusst“ (Michalak et al., 2015) bzw. „sprachaufmerksam“ (Schmölzer-Eibinger, 2011) gestaltet, schließt er die Reflexion über den Sprachgebrauch und die Entwicklung der Sprachbewusstheit ein. Alle Begriffe haben jedoch gemeinsam, dass sie auf die Verknüpfung der fachlichen und sprachlichen Lernziele hinweisen.

\section{Wichtigste Prinzipien}

Im sprachsensiblen Fachunterricht werden (fach-)sprachliche Kompetenzen nicht vorausgesetzt, sondern schrittweise vermittelt. Hierfür ist die Analyse der (fach)sprachlichen Anforderungen nötig, in der - orientiert an den fachlichen Inhalten sowohl die spezifischen Text- und Diskursformen des jeweiligen Unterrichtsfaches als auch die typischen sprachlichen Handlungen wie Beschreiben, Erklären, Protokollieren etc. berücksichtigt werden. Darauf aufbauend werden die fachlichen und sprach- 
lichen Lernziele formuliert und der Erwartungshorizont ausgearbeitet, um mögliche Stolpersteine zu identifizieren. Der sprachsensible Fachunterricht, geplant beispielsweise mit dem Planungsraster von Tajmel und Hägi-Mead (2017), knüpft an den Lernstand der Schüler(innen) an und führt sie durch eine Auswahl von Unterstützungstechniken und Differenzierungsmöglichkeiten an die konzeptionelle Schriftlichkeit heran. Hierbei ist es empfehlenswert, Methoden auszuwählen, welche viel sprachliches Handeln für die Schüler(innen)schaft ermöglichen. Eine Hilfestellung für die Planung bzw. Reflexion des eigenen Fachunterrichts bieten Michalak et al. (2015) mit zehn Prinzipien des sprachbewussten Unterrichts oder Thürmann und Vollmer (2013) mit einer Checkliste für sprachsensible Aspekte des Fachunterrichts.

\section{Literatur}

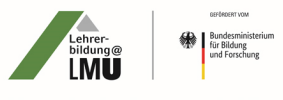

Gogolin, I. \& Lange, I. (2010). Durchgängige Sprachbildung. Eine Handreichung. Münster: Waxmann.

Leisen, J. (2013). Handbuch Sprachförderung im Fach. Stuttgart: Klett.

Michalak, M., Lemke, V. \& Goeke, M. (2015). Sprache im Fachunterricht. Tübingen: Narr.

Riebling, L. (2013). Sprachbildung im naturwissenschaftichen Unterricht. Eine Studie im Kontext migrationsbedingter sprachlicher Heterogenität. München: Waxmann.

Schmölzer-Eibinger, S. (2011). Lernen in der Zweitsprache. Grundlagen und Verfahren der Förderung von Textkompetenz in mehrsprachigen Klassen. Tübingen: Narr.

Tajmel, T. \& Hägi-Mead, S. (2017). Sprachbewusste Unterrichtsplanung. Prinzipien, Methoden und Beispiele für die Umsetzung. FörMig Material, 9. Band. Münster \& New York: Waxmann.

Thürmann, E. \& Vollmer, H. J. (2013). Schulsprache und Sprachsensibler Fachunterricht: Eine Checkliste mit Erläuterungen. In C. Röhner \& B. Hövelbrinks (Hrsg.), Fachbezogene Sprachförderung in Deutsch als Zweitsprache. Theoretische Konzepte und empirische Befunde zum Erwerb bildungssprachlicher Kompetenzen (S. 212233). Weinheim: Beltz.

\section{Weiterführende Literatur}

Ahrenholz, B., Hövelbrinks, B. \& Schmellentin, C. (2017). Fachunterricht und Sprache in schulischen Lehr-/Lernprozessen. Tübingen: Narr.

Kniffka, G. \& Roelcke, Th. (2016). Fachsprachenvermittlung im Unterricht. Paderborn: UTB.

Lütke, B., Petersen, H. \& Tajmel, T. (2017). Fachintegrierte Sprachbildung: Forschung, Theoriebildung und Konzepte für die Unterrichtspraxis. Berlin, Boston: De Gruyter.

Michalak, M., Lemke, V. \& Goeke, M. (2015). Sprache im Fachunterricht. Tübingen: Narr. 
Wildemann, A. \& Fornol, S. (2016). Sprachsensibel unterrichten in der Grundschule: Anregungen für den Deutsch-, Mathematik- und Sachunterricht. Seelze: Klett, Kallmeyer. 